\title{
Percepción de los consumidores turísticos en la prestación de servicios de campamento de los balnearios de Ixmiquilpan, Hidalgo.
}

\section{Tourist consumers perception in the provision of camping services in the spas of Ixmiquilpan, Hidalgo}

\author{
Myriam Coronado-Meneses ${ }^{a}$, Jaime R. Campos-González ${ }^{b}$ Edgar A. Magus-Bustos ${ }^{c}$, Dora L. \\ Fragoso-Manzo $^{d,}$ Maribel Navarro-Gálvez ${ }^{e}$, Adriana G. Villalobos-Sánchez ${ }^{f}$
}

\begin{abstract}
:
The present work, which identifies the perception of tourist consumers in the provision of camping services in the resorts of Ixmiquilpan, Hidalgo, is constituted in two stages and responds to recommendations of federal guidelines, and the Official Mexican Standard NOM-06-TUR-2009 regarding the quality of tourism, hygiene and safety services.

The results of the research instrument applied to tourists indicate that there are compliance factors, on the part of service providers, which have a positive impact on the transmission of guidelines; however, there are regulations on which more emphasis should be placed, mainly to ask tourists for medical or pre-written information from a doctor, if any; information from close relatives to whom to alert in the event of accidents; and to define and determine the area where the camps are located; the foregoing, in order to contribute to the scope of the rule.
\end{abstract}

\section{Keywords:}

Tourist Corridor, Tourist Consumer, Camps, Tourist Standardization

\section{Resumen:}

El presente trabajo, que identifica la percepción de los consumidores turísticos en la prestación de servicios de campamento en los balnearios de Ixmiquilpan, Hidalgo, se constituye en dos etapas y responde a recomendaciones de lineamientos federales, estatales y a la Norma Oficial Mexicana NOM-06-TUR-2009 respecto a la calidad de servicios turísticos, de higiene y de seguridad.

Los resultados del instrumento de investigación aplicado a los turistas indican que hay factores de cumplimiento, por parte de los prestadores de servicio, que inciden positivamente en la transmisión de lineamientos; no obstante, hay reglamentos en los que se debe poner mayor énfasis, principalmente en solicitar a los turistas información médica o pre escrita por un médico, en su caso; información de parientes cercanos a quien avisar en caso de accidentes y en delimitar y determinar la zona en donde se encuentren establecidos los campamentos; lo anterior, para coadyuvar al alcance de la norma.

\section{Palabras Clave:}

Corredor turístico, Consumidor turístico, Campamentos, Normalización Turística.

Universidad Autónoma del Estado de Hidalgo, Escuela Superior de Tizayuca, https://orcid.org/0000-0003-1171-516X, Email: myriam_coronado3187@uaeh.edu.mx

b Universidad Autónoma del Estado de Hidalgo, https://orcid.org/0000-0001-8292-5944, Email: ca392404@uaeh.edu.mx

c Universidad Autónoma del Estado de Hidalgo, https://orcid.org/0000-0002-0443-2017, Email: ma393568@uaeh.edu.mx

d Universidad Autónoma del Estado de Hidalgo, https://orcid.org/0000-0002-6645-3219, Email: dora_fragoso7504@uaeh.edu.mx

e Universidad Autónoma del Estado de Hidalgo, https://orcid.org/0000-0001-5723-0817, Email: maribel_navarro4807@uaeh.edu.mx

f Universidad Autónoma del Estado de Hidalgo, https://orcid.org/0000-0002-7773-9407, Email: adriana villalobos9127@uaeh.edu.mx 


\section{Introducción}

\section{Planteamiento del problema}

El Plan Nacional de Desarrollo (PND) del periodo actual (2019 - 2024) indica en el apartado dos, sobre Políticas Sociales, que se debe priorizar el "Desarrollo sostenible". De igual forma, el Plan señala que el quehacer nacional en su conjunto económico, político, social y cultural no debe ser orientado a multiplicar de manera irracional la producción, la distribución y el consume. En este contexto, se debe considerar, de acuerdo a las Normas Oficiales de Turismo, que se debe procurar el buen manejo de la información del consumidor que garanticen la calidad de los servicios en higiene y seguridad.

Por su parte, el Plan Estatal de Desarrollo del Estado de Hidalgo del periodo actual (2016-2022) reconoce en su eje dos, objetivo dos, estrategia cuatro, que se debe consolidar la oferta de servicios turísticos existentes.

Por último, el documento de creación de la Licenciatura en Turismo indica en su Perfil de egreso que se deben desarrollar competencias en los estudiantes que les posibiliten aplicar conocimientos en técnicas de investigación de mercados y conocer el entorno turístico con relación a las tendencias del mercado.

\section{Justificación}

La realización de esta investigación tuvo diversos motivos que la justifican:

El primero es que los objetivos nacionales indican que el quehacer nacional, no debe ser orientado solo a multiplicar la producción, la distribución y el consumo; aunado a ello, el Comité Consultivo Nacional de Normalización Turística emite Normas Oficiales Mexicanas que linean requisitos mínimos de información, higiene y seguridad que deben cumplir los prestadores de servicios turísticos para garantizar la calidad en el servicio a sus consumidores.

Por lo anterior, se exhibe que el Ejecutivo Federal considera en toda circunstancia los impactos que tendrán sus políticas y programas en el tejido social, en la ecología y en los horizontes políticos y económicos del país. De igual forma, a nivel estatal se busca consolidar la oferta de servicios turísticos existentes.

Por lo anterior, existe la inquietud de conocer si las áreas turísticas denominadas "Balnearios" cuentan con programas de señalamientos y aplicación de reglamentos necesarios respecto al servicio de campamento que brindan a los turistas y si estos tienen conciencia de los aspectos legales con base a la aplicación de estrategias de cuidado y trabajo para un bienestar social, económico y ambiental de acuerdo a la NOM-06-TUR-2009.

Este estudio se justifica porque responde a lo que objetivos nacionales y estatales pretenden alcanzar en materia de seguridad, información y calidad en el servicio.

\section{Objetivo General}

Identificar la percepción de los consumidores turísticos en la prestación de servicios de campamento en los balnearios de Ixmiquilpan, Hidalgo.

\section{Materiales y métodos}

Para cumplir con el objetivo de este trabajo, se optó por un diseño no experimental de tipo transversal con alcance descriptivo.

En la primera etapa se realizó investigación documental con diversas fuentes de información, entre las que se encuentran servidores de información del Instituto Nacional de Estadística y Geografía e Informática (INEGI) así como páginas oficiales de Gobierno de México esto con la finalidad de darle fundamentación a esta investigación.

En la segunda etapa se diseñó un instrumento de investigación y se presenta información primaria con base al análisis de resultados de su aplicación, en el mes de septiembre de 2021.

\section{Instrumentos y materiales}

La investigación utiliza como instrumentos y técnicas las encuestas; en la investigación puede utilizarse la entrevista estructurada y la no estructurada, análisis de documentos, archivos, cuestionarios entre otros.

En cuanto al diseño del instrumento, para alcanzar la finalidad y meta de acuerdo a la propuesta de Brown (1980, p. 21) que comprende etapas que pueden variar su orden, llevarse a cabo varias de manera simultánea u omitir algunas, siendo posible, identificar una secuencia general en los pasos que incluye.

\section{Población y muestra.}

En atención al tema y al objetivo de investigación, la población se constituye, por turistas de los balnearios que integran la Asociación de Balnearios y Parques Acuáticos 
del Estado de Hidalgo en el Corredor de Balnearios de Ixmiquilpan.

Hernández, Fernández y Baptista (2010) definen como unidades de análisis a los individuos u organizaciones que son sujetos de estudio. Con base a ello y para llevar a cabo la investigación de campo de acuerdo a las limitaciones temporales y espaciales del estudio, el instrumento de investigación se aplicó a la muestra de visitantes y turistas

Para determinar el tamaño de la muestra en poblaciones finitas se adoptaron las recomendaciones de Castañeda, De la Torre, Morán y Lara (2002) y se optó por tolerar un porcentaje de error del $4 \%$ con un nivel de confianza del 95\% para aplicar en la ecuación propuesta por los autores.

\section{Apartado I. Marco Conceptual}

\subsection{Corredor turístico.}

Son vías de conexión entre las zonas, las áreas, los complejos, los conjuntos, los atractivos turísticos, los puertos de entrada del turismo receptivo y las plazas emisoras del turismo interno, que funcionan como el elemento estructurador del espacio turístico (Staff, 2016).

\subsection{Atractivo turístico.}

Atractivo turístico es el conjunto de elementos materiales y/o inmateriales susceptibles de ser transformados en un producto turístico que tenga capacidad para incidir sobre el proceso de decisión del turista provocando su visita a través de flujos de desplazamientos (Rivera, 2016).

\subsection{Consumidor turístico}

Entre los principales actores de la actividad turística está el consumidor turístico, a quien Calero (2012) lo define como la persona que se encuentra lejos de su entorno habitual cuya finalidad principal del viaje no es la de ejercer una actividad económica, más bien es la de generar un gasto por el consumo de productos.

\subsection{NOM-06-TUR-2009}

En cumplimiento a lo previsto en el artículo 46, fracción I, de la Ley Federal sobre Metrología y Normalización, el Comité Consultivo Nacional de Normalización Turística aprobó la Norma Oficial Mexicana NOM-06-TUR-2009, que consiste en los requisitos mínimos de información, higiene y seguridad que deben cumplir los prestadores de servicios turísticos de campamentos (DOF, 2009).

\subsection{Campamento}

Se define aquel en que los participantes salen de las zonas urbanas, de su región de origen, o de la civilización y disfrutan de la naturaleza mientras pasan una o varias noches al aire libre en medio de una atmósfera relajada, sin horarios estrictos y sin demasiadas obligaciones (Turismeproactiu, 2018)

\section{Apartado II. Marco Legal.}

La NOM-06-TUR-2009 hace mención de requisitos mínimos de información, higiene y seguridad que deben cumplir los prestadores de servicios turísticos de campamentos.

Principales disposiciones generales de operación:

5.1.2 El Reglamento Interno, debe estar a la vista sin ningún obstáculo (no debe ser obstruido por muebles, columnas, paredes, ramas, árboles, entre otros) en la entrada a las instalaciones, o bien en la casa club, caseta de entrada u otro tipo de instalación similar que su función sea de atención, información, recepción y registro del turista.

5.1.3 El Reglamento Interno, debe aplicarse por el personal del campamento y vigilar que se lleve a cabo por el turista.

5.1.4 El prestador de servicios turísticos en la contratación o durante la prestación del servicio y según las actividades que ofrezca, debe solicitar a cada turista, el llenado de un formato con membrete, cuya información debe contener:

a) Fecha de inicio y término de la estancia en el campamento (descrito por el prestador)

b) Tipo de actividades a desarrollar

Datos proporcionados por el turista

c) Nombre completo, dirección, teléfono, nacionalidad, edad;

d) Padecimientos físicos, alergias, tipo de sangre y en caso de ser niños la dosis de medicamento indicada por un médico;

e) Nombre y teléfono del médico familiar;

f) Nombre, dirección y teléfono de dos parientes cercanos a quien se pueda llamar en caso de accidente.

5.1.8 Cuando el turista lleve consigo personal médico de apoyo, así como perro guía para invidentes, se le debe informar, en su caso, sobre las tarifas adicionales, cuando éstos signifiquen necesariamente un costo extra para otorgar el servicio.

\subsection{De la información}

5.2.1 Los prestadores de servicios turísticos deben informar lo siguiente en sus establecimientos:

5.2.1.1 Las tarifas y características de cada uno de los servicios, así como a lo que tiene derecho, al momento de su admisión y registro.

5.2.1.2 Exhibir la tarifa de los principales servicios ofrecidos y de los servicios adicionales, con caracteres claramente legibles. 
5.2.1.3 Respetar la tarifa pactada, en los casos en que el servicio haya sido reservado con anticipación.

5.2.1.3.1 En caso de cobrar anticipos a los turistas, si así lo establece su política de reservación, éstos deben ser tomados a cuenta de la totalidad del servicio.

5.2.1.4 Entregar al turista al momento de su admisión y registro la factura, recibo o comprobante, en el que consten los datos específicos del servicio contratado, incluyendo anticipos.

\subsection{De las instalaciones}

5.3.1 Delimitar y determinar la zona en donde se encuentren establecidos los campamentos a fin de que los turistas puedan identificarla plenamente.

5.3.2 Señalizar de manera pictográfica y en su caso textual los accesos, rutas de evacuación, veredas y/o senderos, servicios, equipo de emergencia y vigilancia, tomando como base las señales de Protección Civil establecidas por la Secretaría de Gobernación en la NOM003-SEGOB-Vigente.

5.3.2.1 La señalización de los servicios e instalaciones que no estén incluidos en la prevista por protección civil, se debe realizar mediante un análisis previo, tomando en cuenta las condiciones del lugar y considerar que:

a) Las señales informativas se colocan en el lugar preciso en donde las personas tengan tiempo suficiente para captar el mensaje;

b) Las señales preventivas se colocan en donde las personas las puedan observar con tiempo suficiente sin correr riesgo;

c) Las señales prohibitivas se deben colocar en el punto mismo donde exista la restricción.

\section{Apartado III. Los balnearios en Ixmiquilpan, Hidalgo.}

La zona de balnearios en Ixmiquilpan, Hidalgo, tiene múltiples parques acuáticos y zonas de relajación, cuenta con aguas termales que la hacen mucho más atractiva, incluso, en temporadas invernales; no solo por el turismo nacional, sino también por el turismo internacional, siendo este uno de los lugares más populares y de mayor importancia para el turismo en el Estado de Hidalgo (Ixmiquilpan, s.f).

El corredor de Balnearios de Ixmiquilpan se compone de la siguiente oferta turística:

Balneario "Valle Paraíso"

Parque Acuático "El Tephé"

Parque Acuático "Pueblo Nuevo"

Parque Acuático "Te-Pathé"

Parque Acuático "Dios Padre"

Balneario "Dauthi"

Parque Acuático "Tollán”

\author{
Parque Acuático "Maguey Blanco" \\ Parque Ecológico "EcoAlberto"
}

Esta investigación se lleva a cabo en los balnearios que aglutina el municipio de Ixmiquilpan y se tomarán en cuenta específicamente aquellos que brindan servicio de campamentos dentro de sus instalaciones en donde han pernoctado los turistas encuestados para el desarrollo de esta investigación.

\section{Resultados.}

Este apartado presenta resultados que favorecen el alcance del objetivo de investigación y presenta información primaria que brindaron turistas que realizaron campamento en los municipios que implica el trabajo y que, en conjunto, permitieron obtener una visión general para identificar la percepción de los consumidores turísticos en la prestación de servicios de campamento en los balnearios de Ixmiquilpan, Hidalgo, en atención a principales lineamientos de la Norma Oficial Mexicana NOM-06-TUR-2009.

El $81.5 \%$ de los turistas hicieron mención que visualizaron sin ningún obstáculo el reglamento interno, no obstante, el $18.5 \%$ hace mención de la deficiencia de este servicio.

El $84.2 \%$ menciono que las empresas turísticas fueron claras al solicitarles el llenado de formato de estancia en campamento, por otra parte, el $15.8 \%$ de los turistas menciono una falta de información para su estancia.

Los turistas mencionaron que no les solicitan información médica (81.8\%) solo el $18.2 \%$ mencionó que si les solicitaron información médica adicional o pre escrita por un médico

Los turistas exhibieron que, en cuanto a información adicional de parientes cercanos en caso de accidente, solo se lo solicitaron al $50 \%$ de ellos, mientras el otro $50 \%$ dice no haberles solicitado dicha información.

En cuestión a la información sobre tarifas adicionales, el $96.6 \%$ de los turistas mencionaron que, si estaban disponibles, mientras que un $3.4 \%$ mencionó que no se les hizo mención de dichas tarifas de servicio.

Un $80 \%$ de los turistas menciono que sí les informaron de manera correcta los servicios y derechos al momento de su admisión y registro, no obstante, el $20 \%$ restante hace mención a una ausencia de dichos señalamientos.

$78.3 \%$ de los turistas mencionaron que si les entregaron factura y/o recibo mientas que el $21.7 \%$ de ellos exhibieron que no se les proporciono algún recibo por sus respectivos pagos.

El $76.2 \%$ de turistas exhibieron una buena visualización de las rutas de evacuación veredas y/o senderos, servicios, equipo de emergencia y vigilancia, mientras que 
el 23.8\% menciono que hay una deficiencia en cuestión a la señalización.

El $73.4 \%$ de los turistas mencionaron que, a lo largo del complejo, les fue factible delimitar y determinar la zona en donde se encuentren establecidos los campamentos y el $26.6 \%$ de ellos no lo pudo identificar plenamente.

Los turistas mencionan que a lo largo de los complejos los señalamientos de prohibición son visibles esto lo menciona un $88.9 \%$, por otra parte, el $11.1 \%$ de los visitantes menciona no encontrar o visualizar dichos señalamientos

El $90 \%$ de los turistas exhibió poder visualizar con claridad y sin interrupción las medidas de seguridad de las albercas, por otra parte, el $10 \%$ de los turistas menciona dificultad para visualizar el reglamento de uso de las albercas.

\section{Conclusiones}

Los resultados de la aplicación de instrumento de investigación conducen a las siguientes conclusiones:

Los balnearios del municipio de Ixmiquilpan cumplen en su mayoría con los lineamientos que dicta la NOM-06TUR-2009 respecto a:

- Visualizaron del reglamento interno

- Llenado de formato de estancia en campamento

- Conocimiento de tarifas adicionales, servicios y derechos al momento de su admisión y registro

- Visualización de las rutas de evacuación veredas y/o senderos, servicios, equipo de emergencia y vigilancia

- Señalamientos de prohibición

- Visualización de medidas de seguridad de las albercas

Sin embargo, es importante que los balnearios brinden mayor importancia en los siguientes apartados legales:

- Solicitar a los turistas el llenado de información médica adicional o pre escrita por un médico, en su caso.

- Solicitar nombre, dirección y teléfono de dos parientes cercanos a quien se pueda llamar en caso de accidente.

- Entrega de factura y/o recibo por los servicios brindados.

- Delimitar y determinar la zona en donde se encuentren establecidos los campamentos

En atención a lo anterior, con el propósito de lograr lo que pretende la NOM sobre la seguridad y la calidad de los servicios que tienen estos establecimientos para con sus consumidores turísticos para mantener un estándar de control de las áreas.

\section{Referencias}

[1] Staff, E. (2016). Entorno Turistico.com. Obtenido de: https://www.entornoturistico.com/explandict/corredor-turistico/.

[2] Rivera, D. (2016). Blogspot. Obtenido de: http://estudiosdelturismo.blogspot.com/2009/05/conceptos-depatrimonio-atractivo.html.

[3] Calero, A. (2012). Comportamiento del Consumidor turistico. México: Prentice Hall.

[4] Comité Consultivo Nacional de Normalización Turística. [CCNNT]. Normas mexicanas. MX. Obtenido de DOF.GOB.MX: http://www.dof.gob.mx/normasOficiales/4770/salud/salud.htm.

[5] Turismeproactiu. (2018). Obtenido de: http://www.turismeproactiu.com/es/por-que-es-popular-el-camping/.

[6] Ixmiquilpan, H. (S/F). Enciclopedia de los Municipios de México Recuperado de: http://intranet.ehidalgo.gob.mx/enciclomuni/municipios/13030a.htm

[7] Poder Ejecutivo Federal. (2019). Plan Nacional de Desarrollo 20072012. Recuperado de https://lopezobrador.org.mx/wpcontent/uploads/2019/05/PLAN-NACIONAL-DE-DESARROLLO2019-2024.pdf

[8] Secretaría de Gobierno. (2016). Plan Estatal de Desarrollo 2016-2022. Recuperado de http://planestataldedesarrollo.hidalgo.gob.mx/

[9] Brown, F. (1980). Principios de la medición en psicología y educación. México: El manual moderno.

[10] Castañeda, J., de la Torre, M., Morán, J., \& Lara, L. (2002). Metodología de la investigación. México: McGraw Hill 